\title{
Anisotropic magnetization, critical temperature, and paramagnetic Curie temperature in the highly anisotropic magnetic Heusler compound $\mathrm{Rh}_{2} \mathrm{CoSb}$
}

\author{
Yangkun He $\odot,{ }^{1, *}$ Romain Sibille $\odot,{ }^{2}$ Dong Chen $\odot,{ }^{1}$ Johannes Kroder, ${ }^{1}$ Toni Helm $\odot,{ }^{1,3}$ Walter Schnelle, ${ }^{1}$ \\ Claudia Felser, ${ }^{1}$ and Gerhard H. Fecher ${ }^{1}$ \\ ${ }^{1}$ Max-Planck-Institute for Chemical Physics of Solids, D-01187 Dresden, Germany \\ ${ }^{2}$ Laboratory for Neutron Scattering and Imaging, Paul Scherrer Institut, 5232 Villigen PSI, Switzerland \\ ${ }^{3}$ Dresden High Magnetic Field Laboratory (HLD-EMFL), Helmholtz-Zentrum Dresden-Rossendorf, 01328 Dresden, Germany
}

(Received 6 April 2021; revised 18 May 2021; accepted 4 June 2021; published 21 June 2021)

\begin{abstract}
The paramagnetic Curie temperature $\theta_{\mathrm{p}}$ is a concept that describes the magnetic ordering temperature in the well-established Curie-Weiss law. Despite the successful explanations of the magnetic behavior, the anisotropy is not usually considered. Although anisotropic $\theta_{\mathrm{p}}$ has been reported for several layered antiferromagnetic or ferrimagnetic materials owing to the orientation-dependent exchange, in ferromagnetic systems, $\theta_{\mathrm{p}}$ was thought to be almost isotropic for decades, and the occasionally reported small difference has remained unexplained. In this paper, we experimentally report the anisotropic magnetization, critical temperature, and paramagnetic Curie temperature in highly anisotropic magnetic $\mathrm{Rh}_{2} \mathrm{CoSb}$ caused by a large magnetocrystalline anisotropy. The saturation magnetization along the $c$ axis is $25 \%$ larger than that along the $a$ axis. The critical temperature and paramagnetic Curie temperature along the $c$ axis are 6 and $15 \mathrm{~K}$ higher than those along the $a$ axis, respectively, as deduced from the Arrott plots and inverse susceptibility. A simple modification of the Curie-Weiss law was made to calculate the anisotropic $\theta_{\mathrm{p}}$, which well explains not only $\mathrm{Rh}_{2} \mathrm{CoSb}$, but also many other previously reported ferromagnetic materials.
\end{abstract}

DOI: 10.1103/PhysRevB.103.214436

\section{INTRODUCTION}

The magnetocrystalline anisotropy $K_{1}$ is the energy that forces the moments to align along the easy axis. Various phenomena related to this energy in magnetism, such as magnetostriction [1], hard magnetism [2], and anisotropic magnetoresistance [3], have been studied and applied in our daily lives. However, there are also several less well-known effects related to $K_{1}$, including anisotropic magnetization $[4,5]$, critical temperature, and paramagnetic Curie temperature $[6,7]$.

The magnetization along the easy axis is larger than that along the hard axis because, under the effect of both thermal energy and $K_{1}$, the moments lie in a narrow cone along the easy axis, whereas a wider cone appears for magnetization along the hard axis [4]. The difference in magnetization along both axes is negligible in ordinary $3 d$ ferromagnets. For example, the ratios of anisotropic magnetization or saturation magnetization (anisotropic magnetization ratio $\Delta m / m$ ) are $\sim 0.01 \%$ for $\mathrm{Fe}$ and $\mathrm{Ni}$ [8]. However, in highly anisotropic

\footnotetext{
*yangkun.he@cpfs.mpg.de
}

Published by the American Physical Society under the terms of the Creative Commons Attribution 4.0 International license. Further distribution of this work must maintain attribution to the author(s) and the published article's title, journal citation, and DOI. Open access publication funded by the Max Planck Society. systems, the values are much larger: $4 \%$ for $\mathrm{YCo}_{5}[9,10], 5 \%$ for $\mathrm{MnBi}[11,12]$, and $9 \%$ for $\mathrm{Fe}_{2} \mathrm{P}$ [13].

A ferromagnet loses its spontaneous long-range-ordered moment at the Curie temperature, above which thermal energy overcomes the magnetic interactions, leading to paramagnetism. However, the concept of Curie temperature is only valid along the easy axis in whose direction the moments spontaneously align at the zero field. Applying a magnetic field enables the moments to be forcibly aligned away from the easy axis or even along the hard axis with the magnetic ordering below a critical temperature $\left(T_{c}\right)$. Generally, for most magnetic materials, the critical temperature is similar to the Curie temperature. However, being "similar" is not equivalent to being the "same." Callen reported in a theoretical work that a large $K_{1}$ and a small exchange energy can lead to an anisotropic critical temperature [6]. Thereafter, ferrimagnetic $\mathrm{Fe}_{7} \mathrm{~S}_{8}$ was reported to be paramagnetic along the $c$ axis and ferromagnetic on the $a b$ plane [7], in agreement with the theoretical prediction. However, little evidence has been found in most ferromagnetic systems.

The paramagnetic Curie temperature $\theta_{\mathrm{p}}$ is another concept for describing the magnetic ordering temperature by fitting the inverse susceptibility in the paramagnetic state using the Curie-Weiss law, which is usually higher than the Curie temperature. Above $\theta_{\mathrm{p}}$, the short-range-ordered magnetic structures vanish. Despite the successful explanation of the magnetic behavior, the anisotropy is not considered in the Curie-Weiss law. However, it was found that, in several layered antiferromagnets (e.g., $\mathrm{TbRh}_{2} \mathrm{Si}_{2}$ [14]) and ferrimagnets (e.g., $\left.\mathrm{Fe}_{7} \mathrm{~S}_{8}[7]\right), \theta_{\mathrm{p}}$ can be significantly different between the 
(a)

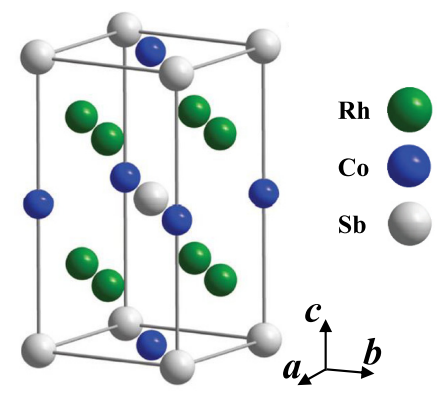

(d)

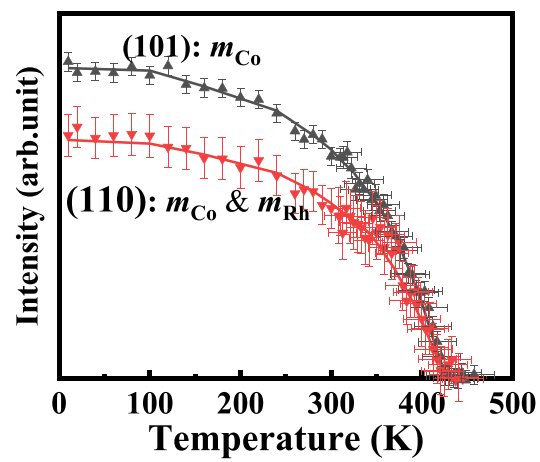

(b)

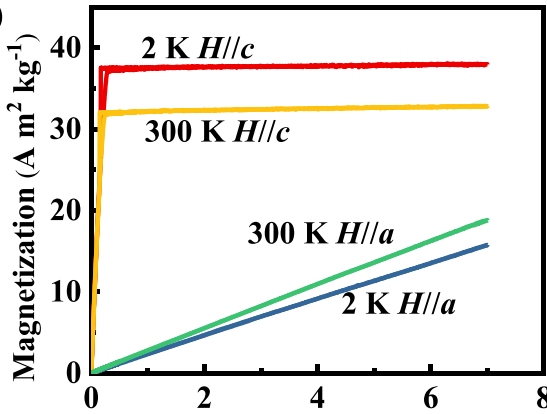

(e)

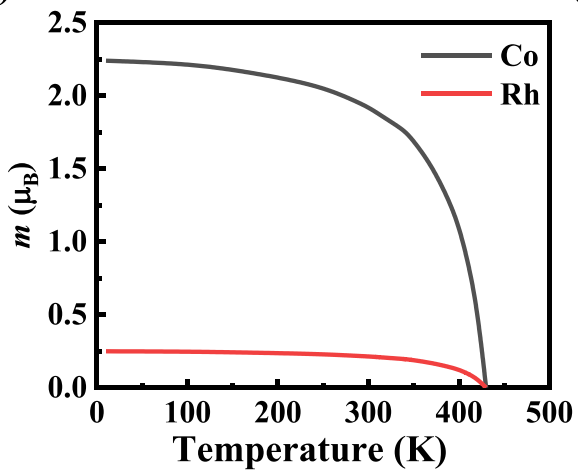

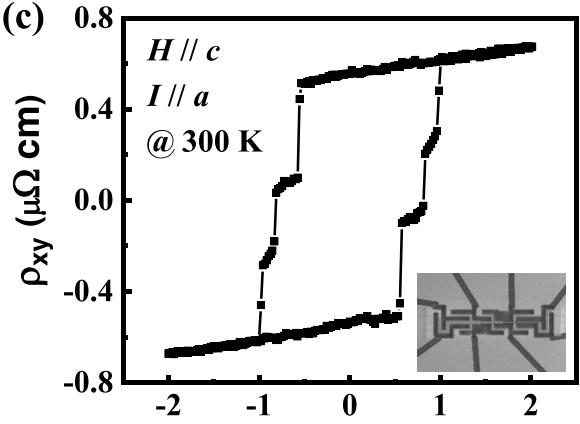

(f)

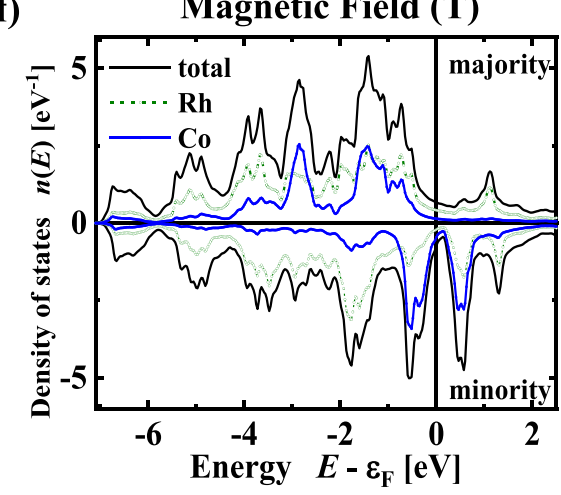

FIG. 1. Hard magnetic properties of $\mathrm{Rh}_{2} \mathrm{CoSb}$. (a) Crystal structure. (b) Magnetization curves along the $c$ and $a$ axes at 2 and $300 \mathrm{~K}$. (c) Hall resistivity of a single crystal cut by a focused iron beam (FIB) with a width of $1.37 \mu \mathrm{m}$ and a thickness of $1.16 \mu \mathrm{m}$. The inset shows the image of the FIB sample. The large coercivity of approximately $1 \mathrm{~T}$ determined from the anomalous Hall effect (c) is indicative for the large $K_{1}$. (d) Intensities of the (101) and (110) peaks of the neutron diffraction. The intensity of the (101) peak is only related to the Co moment, whereas the intensity of the (110) peak is related to both the Co and the Rh moments. The solid curves are the intensities for both magnetic Bragg reflections calculated to provide good agreement with the overall temperature dependence and assuming that both the Co and the Rh moments follow the same order parameter. (e) Temperature dependence of the magnetic moment value. (f) Calculated density of states. Rh is spin polarized.

hard and easy axes even with different signs. This is explained by the ferromagnetic in-plane exchange interaction and antiferromagnetic coupling between the layers along the $c$ axis.

Compared with antiferromagnetic systems where anisotropic exchange interactions play a decisive role in $\theta_{\mathrm{p}}$, ferromagnetic systems usually have ferromagnetic interactions in all directions. Therefore, $\theta_{\mathrm{p}}$ was thought to be almost isotropic for decades and the occasionally reported small difference has remained unexplained $[15,16]$. In theory $[6,17,18]$, a large anisotropic $\theta_{\mathrm{p}}$ in ferromagnetic materials can also be realized in systems with a large crystal field, although only a few reports exist.

$\mathrm{Rh}_{2} \mathrm{CoSb}$ is a tetragonal Heusler crystallized in the $D_{22}$ structure with $a=4.0393(6)$ and $c=7.1052(7) \AA$. The $4 d$ $\left(0 \frac{1}{2} \frac{1}{4}\right)$ site is occupied by Rh, $2 b\left(00 \frac{1}{2}\right)$ by $\mathrm{Co}$, and $2 a(0$ $00)$ by $\mathrm{Sb}$ as shown in Fig. 1(a). $\mathrm{Rh}_{2} \mathrm{CoSb}$ is a hard magnet with a $K_{1}$ of $3.6 \mathrm{MJ} \mathrm{m}^{-3}$ and a Curie temperature of around $450 \mathrm{~K}$ [19]. The anisotropy field is $17.5 \mathrm{~T}$ at $2 \mathrm{~K}$ and $12 \mathrm{~T}$ at $300 \mathrm{~K}$ obtained from extrapolation as shown in Fig. 1(b). The magnetic hardness parameter is $\kappa=4.1$ at $2 \mathrm{~K}$ and 3.7 at $300 \mathrm{~K}$, which is the largest among all rare-earth free magnets that exhibit saturation magnetization $\mu_{0} M_{\mathrm{s}}>0.4 \mathrm{~T}$. The highly anisotropic magnetic properties of $\mathrm{Rh}_{2} \mathrm{CoSb}$ provide a good opportunity to study the anisotropic magnetization, critical temperature, and paramagnetic Curie temperature in this system.
In this paper, we report the anisotropic magnetization, critical temperature, and paramagnetic Curie temperature in single crystals of ferromagnetic $\mathrm{Rh}_{2} \mathrm{CoSb}$. A large anisotropic magnetization ratio of $25 \%$ is observed. The anisotropic critical temperature and paramagnetic Curie temperature are dominated by the crystal field, which can be estimated using magnetocrystalline anisotropy.

\section{EXPERIMENTAL DETAILS}

Single crystals were grown using the Bridgeman method [19]. The composition of the crystals was determined by
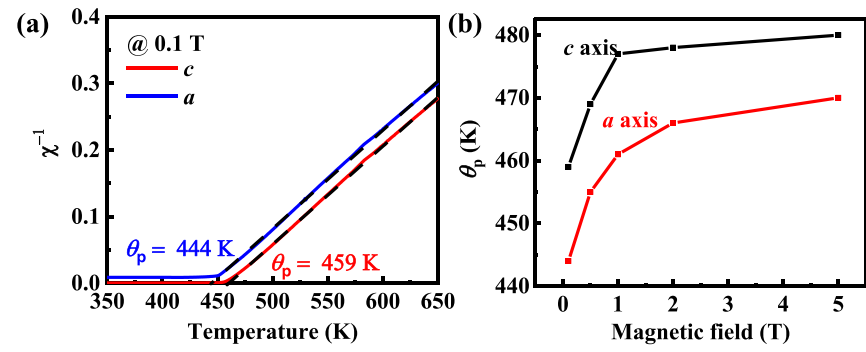

FIG. 2. (a) Temperature dependence of inverse susceptibility along both the $c$ and the $a$ axes. (b) Magnetic-field dependence of $\theta_{\mathrm{p}}$ along the $c$ and $a$ axes. 

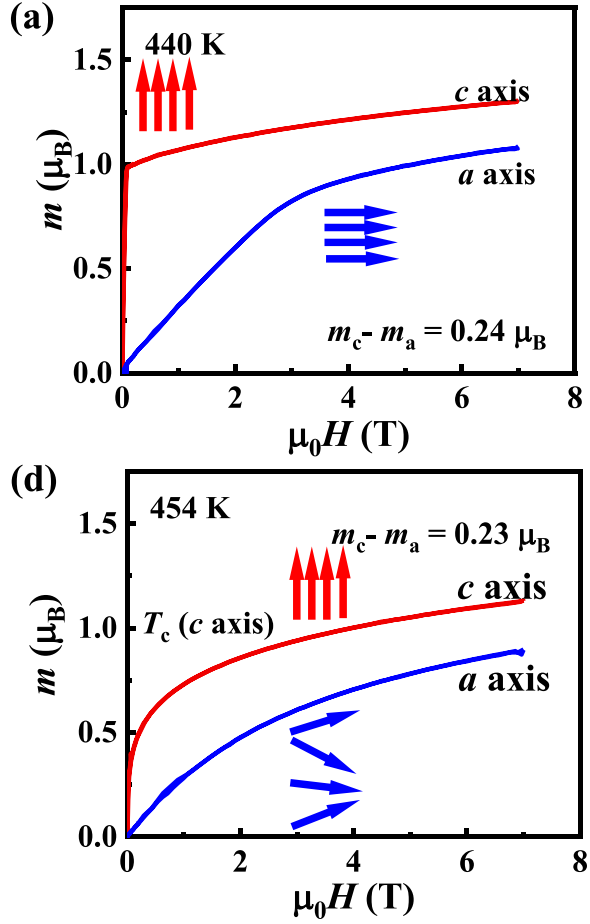
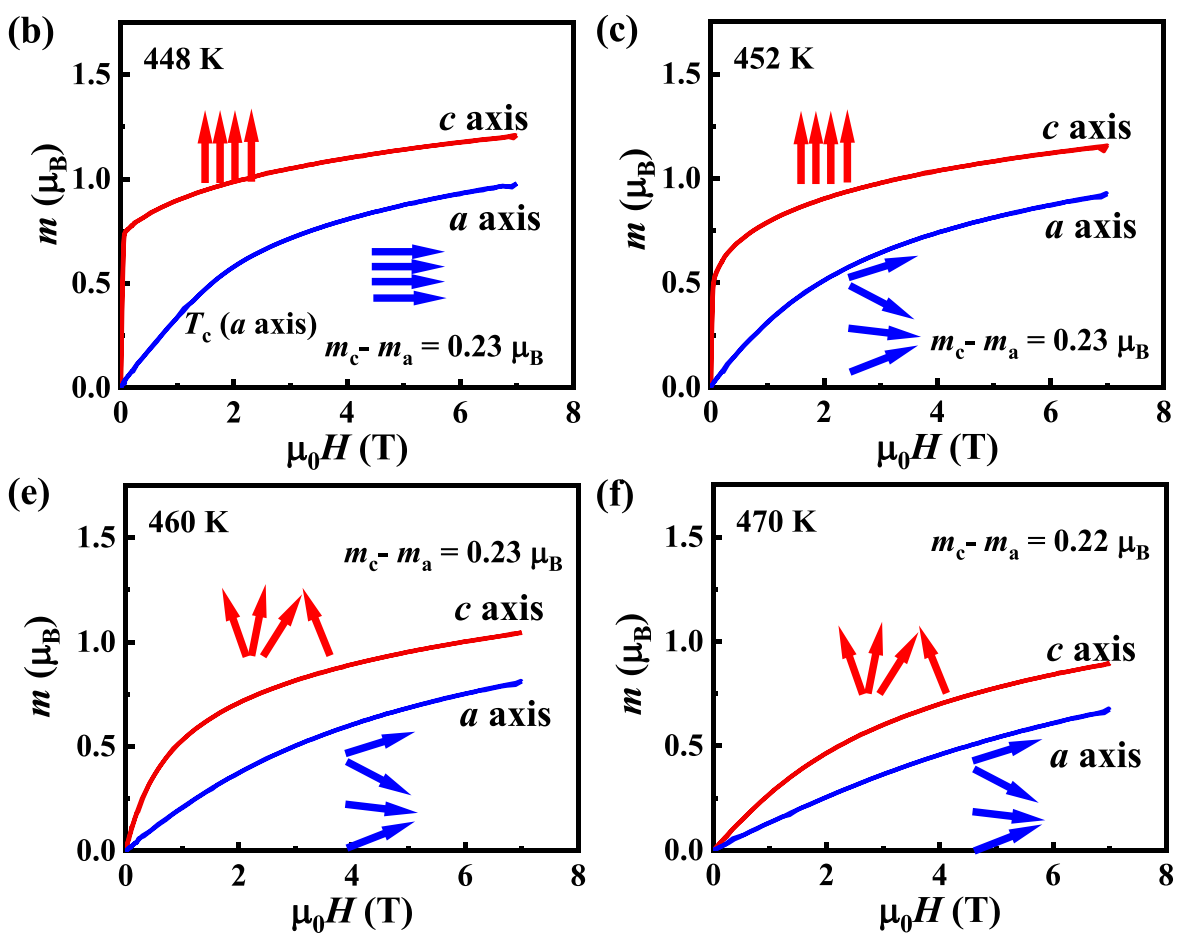

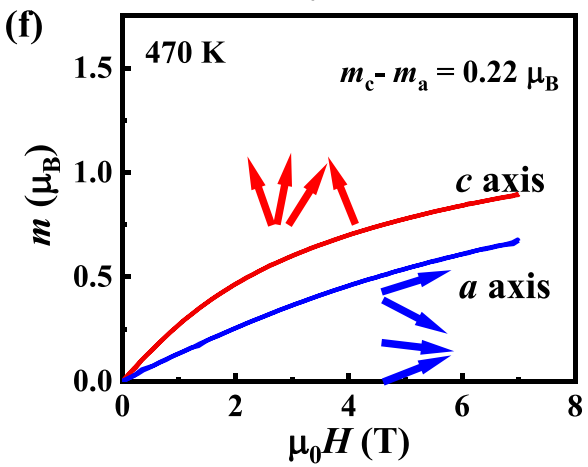

FIG. 3. Magnetization curves along the $c$ and $a$ axes around the Curie temperature. The inserted (non)parallel arrows schematically sketch the long-range (dis)ordered magnetic structure.

wavelength dispersive $\mathrm{x}$-ray spectroscopy, which showed a homogenous composition of $\mathrm{Rh}_{50.3} \mathrm{Co}_{25.6} \mathrm{Sb}_{24.1}$. The magnetization was measured on single crystals with a magnetic field applied along either the $a$ or the $c$ axis using a vibrating sample magnetometer (magnetic property measurement system 3, Quantum Design). The sample size was $0.65 \times$ $0.78 \times 1.30 \mathrm{~mm}^{3}$. Single-crystal neutron diffraction was performed on the Zebra instrument at the Swiss Spallation Neutron Source SINQ of the Paul Scherrer Institute in Villigen, Switzerland. The instrument was operated in four-circle geometry using an Eulerian cradle, and the energy of the incoming beam was selected using a $\mathrm{Ge}(311)$ monochromator, resulting in a wavelength of approximately $1.175 \AA$. To cover the entire temperature range, we conducted two experiments on the same sample using a close-cycle refrigerator between 10 and $320 \mathrm{~K}$ and a vacuum furnace operated between 300 and $480 \mathrm{~K}$. About $120 \mathrm{Bragg}$ reflections were measured at fixed temperatures of 10,300 , and $480 \mathrm{~K}$. The integrated intensities were analyzed using the FULLPROF software suite [20] to refine the nuclear and magnetic structures.

\section{RESULTS}

As $\mathrm{Rh}_{2} \mathrm{CoSb}$ is a highly anisotropic material, its coercivity can easily reach $1 \mathrm{~T}$ when the crystal dimension is decreased to $1.37 \mu \mathrm{m}$ as can be seen in the anomalous Hall effect measurements in Fig. 1(c). The total moment of $2.6 \mu_{\mathrm{B}}$ per formula unit is contributed by ferromagnetically coupled $\mathrm{Co}$ and $\mathrm{Rh}$. This was confirmed by our neutron-diffraction study, which obtained ordered moments of $0.25 \pm 0.06 \mu_{\mathrm{B}}$ and $2.24 \pm 0.05 \mu_{\mathrm{B}}$ at $10 \mathrm{~K}$ for $\mathrm{Rh}$ and $\mathrm{Co}$, respectively.
Their temperature dependence was studied by following the intensity of two magnetic Bragg reflections as shown in Fig. 1(d). The reflections were selected to attempt to distinguish the evolution of the two moments. Although the data over the entire temperature range can be accounted for by assuming that $\mathrm{Rh}$ and Co follow the same order parameter [Figs. 1(d) and 1(e)], significant deviations from this idealized behavior cannot be excluded from the statistics of the present experiment. In particular, our calculation [21] in Fig. 1(f) shows that the Co moment is more localized and that the $\mathrm{Rh}$ moment is induced as also inferred from the unquenched Co orbital moment from our previous x-ray magnetic circular dichroism experiment [19]. In this scenario, the coupling between Rh and Co could decrease drastically upon approaching the Curie temperature and as a result $\mathrm{Rh}$ loses its magnetism quickly, which causes the abnormal behavior in the AC susceptibility in addition to the Hopkinson effect $[19,22]$.

We checked the paramagnetic Curie temperatures based on the inverse susceptibility in Fig. 2. For this, we fit temperaturedependent data along both axes using the Curie-Weiss law,

$$
\chi=C /\left(T-\theta_{\mathrm{p}}\right)
$$

where $\chi$ is the susceptibility and $C$ is the Curie constant. When the applied magnetic field is $0.1 \mathrm{~T}$, the values of $\theta_{\mathrm{p}}$ along the $c$ and $a$ axes are $459 \pm 1$ and $444 \pm 1 \mathrm{~K}$, respectively, as shown in Fig. 2(a). The Curie constants for both axes are the same, corresponding to an effective moment of $1.96 \mu_{\mathrm{B}}$, which roughly agrees with the measured ordered moment of $2.6 \mu_{\mathrm{B}}$ at $2 \mathrm{~K}$. Figure $2(\mathrm{~b})$ shows the $\theta_{\mathrm{p}}$ calculated from the data measured with different magnetic fields. Notably, $\theta_{\mathrm{p}}$ along both axes exhibits a field dependence, which 

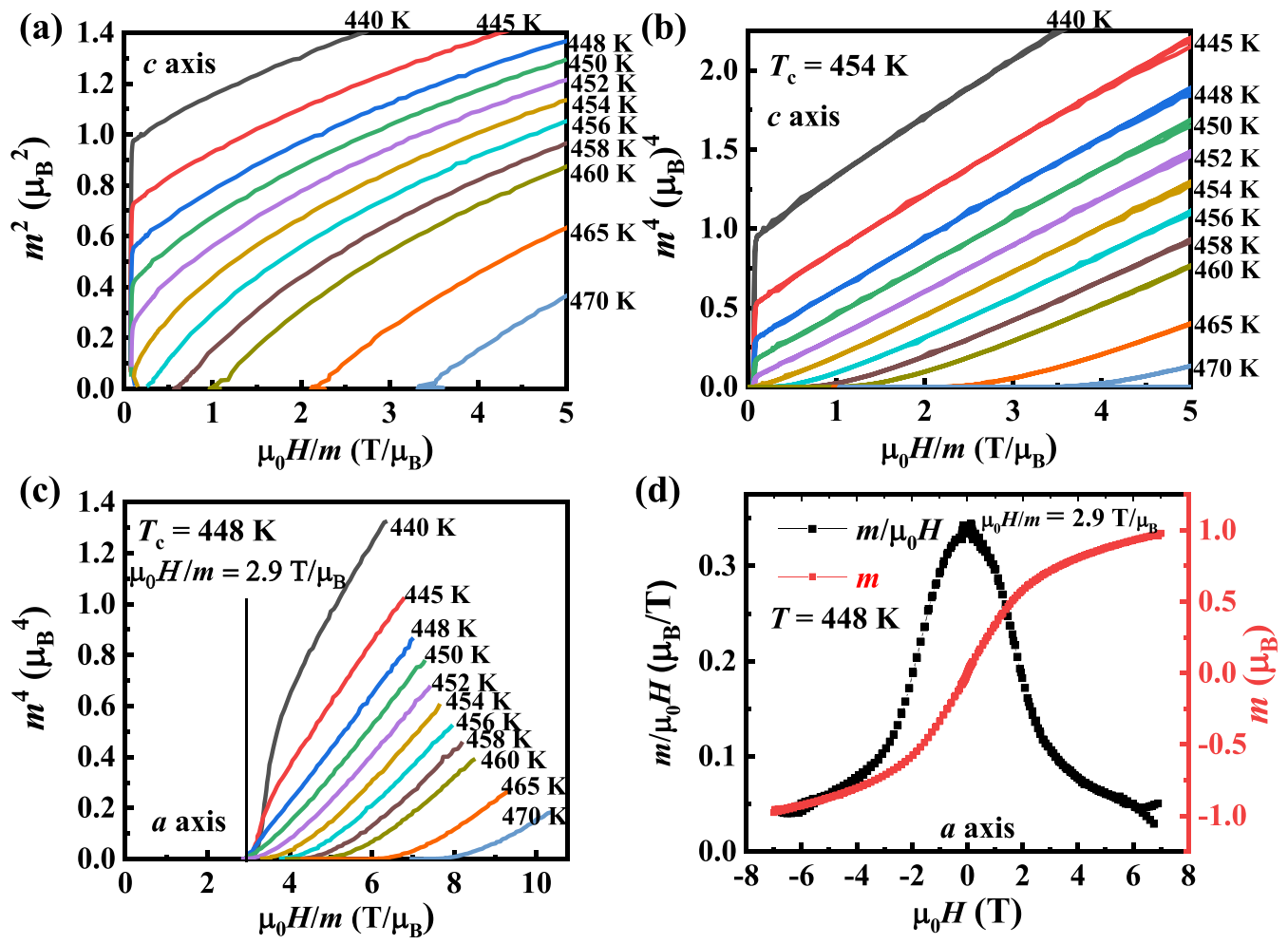

FIG. 4. (a) Arrott curves of $M^{2}$ vs $H / M$ along the $c$ axis. Modified Arrott curves of $M^{4}$ vs $H / M$ along the (b) $c$ and (c) $a$ axes. (d) Susceptibility and magnetization when $H$ was along the $a$ axis at $448 \mathrm{~K}$.

can be explained by the field-dependent susceptibility due to the short-range order when the temperature is not too far from the Curie temperature. Even in the paramagnetic region, the magnetization curve is not linear, and the susceptibility $\chi$ decreases with increasing field. As a result, $\theta_{\mathrm{p}}$ increases with increasing magnetic field. However, the difference between the $c$ and the $a$ axes does not change and the value is always between 12 and $15 \mathrm{~K}$.

The magnetization curves close to the Curie temperature along both the $c$ and the $a$ axes are shown in Fig. 3. The magnetization along the $c$ axis is significantly larger than that along the $a$ axis. The difference is $0.24 \mu_{\mathrm{B}}$ per formula at $440 \mathrm{~K}$ and $7 \mathrm{~T}$, which is about $25 \%$ of the total moment, a value that is much larger than that in $\mathrm{YCo}_{5}(4 \%$ at 4.2 to $300 \mathrm{~K}$ under $20 \mathrm{~T}[9,10]), \mathrm{MnBi}(5 \%$ at $5 \mathrm{~K}$ under $6 \mathrm{~T}$ $[11,12])$, and $\mathrm{Fe}_{2} \mathrm{P}$ (9\% at $5 \mathrm{~K}$ under $\left.7 \mathrm{~T}[13]\right)$. The anisotropic crystal fields together with the large spin-orbit coupling, are responsible for highly anisotropic magnetization. The difference in magnetization along the $c$ and $a$ axes decreases with increasing temperature, although it still exists in the paramagnetic state as shown in Fig. 3(f). The first impression on critical temperatures can be obtained by the bare eye when considering the $M(H)$ magnetization curves at different temperatures. Below $454 \mathrm{~K}$, the magnetization curves along the $c$ axis saturate (in a single-domain state) rapidly as shown in Figs. 3(a)-3(d). At higher temperatures [Figs. 3(e) and 3(f)], the initial susceptibility decreased and the magnetization was a bent curve. Therefore, the critical temperature for the $c$ axis (also the Curie temperature) was around $454 \mathrm{~K}$. The magnetization curve along the $a$ axis is linear before saturation at $440 \mathrm{~K}$, and it saturates at approximately $3.2 \mathrm{~T}$. However, above $452 \mathrm{~K}$, the curve is no longer linear at low field, indicating that the critical temperature along the $a$ axis is lower than $452 \mathrm{~K}$.

The Arrott curves of $M^{2}$ vs $H / M$ were used to precisely determine the critical temperature $T_{c}$ for $H$ along the $c$ axis as shown in Fig. 4(a). In theory, the high-field curves should be a series of parallel straight lines in the high-field range, and the intercept of $M^{2}$ is positive below $T_{c}$ and negative above $T_{c}$. However, all the curves in this plot are nonlinear with a downward curvature even in the high-field region, which indicates that the long-range Landau mean-field theory is not satisfied for $\mathrm{Rh}_{2} \mathrm{CoSb}$. Instead, a tricritical mean-field model $\left(M^{4}\right.$ vs $\left.H / M\right)[23,24]$ is used, and a good fit is obtained as shown in Fig. 4(b). In this model, where the critical exponents $\beta, \gamma$, and $\sigma$ are $0.25,1$, and 5 , respectively, the magnetic phase transition is three dimensional, indicating a nearly isotropic exchange interaction. The $T_{c}$ of the $c$ axis was determined to be $454 \pm 1 \mathrm{~K}$.

Similarly, a tricritical mean-field model was also used to determine $T_{c}$ for the $a$ axis as shown in Fig. 4(c). The $a$ axis is the hard axis, and the initial susceptibility $\chi_{0}$ is only $0.35 \mu_{\mathrm{B}} \mathrm{T}^{-1}$ [Fig. 4(d)], which is far smaller than that along the $c$ axis. Therefore, the intercept on $\chi_{0}{ }^{-1}\left(2.9 \mathrm{~T}_{\mathrm{B}}{ }^{-1}\right)$ was used to determine $T_{c}$ rather than zero along the $c$ axis. This method is also used in polycrystalline samples, whose $\chi_{0}{ }^{-1}$ values are also far from zero [25]. The modified Arrott curves $\left(M^{4}\right.$ vs $\left.H / M\right)$ showed a $T_{c}$ of $448 \pm 1 \mathrm{~K}$. This value is $6 \mathrm{~K}$ below that for the $c$ axis, which is not due to the inaccuracy of the measurement. 


\section{DISCUSSION}

According to a previous theory $[17,18]$, the values of $\theta_{\mathrm{p}}$ along the $c$ and $a$ axes (i.e., $\theta_{\mathrm{p}}^{c}$ and $\theta_{\mathrm{p}}^{a}$, respectively) are determined by both the exchange and the crystal field as follows:

$$
\begin{aligned}
\theta_{p}^{c} & =-\frac{J(J+1)}{3 k_{B}} J_{e x}^{c}-\frac{(2 J-1)(2 J+3)}{5 k_{B}} B_{2}^{0}, \\
\theta_{p}^{a} & =-\frac{J(J+1)}{3 k_{B}} J_{e x}^{a}+\frac{(2 J-1)(2 J+3)}{10 k_{B}} B_{2}^{0},
\end{aligned}
$$

where $B_{2}^{0}$ is a crystal-field parameter; $J$ is the total angular moment of the atom; $J_{e x}^{c}$ and $J_{e x}^{a}$ are the sum over all exchange constants along the $c$ and $a$ axes, respectively; and $k_{\mathrm{B}}$ is the Boltzmann constant. The first term is related to the exchange, whereas the second term is related to the crystal field. These two equations have already been proven to work in antiferromagnetic systems $[14,26]$ dominated by the anisotropic exchange. In $\mathrm{Rh}_{2} \mathrm{CoSb}$, assuming that $J_{e x}^{c} \approx J_{e x}^{a}$ for a ferromagnetic interaction (from the tricritical mean-field model in Fig. 4), we can deduce the theoretical difference in $\theta_{\mathrm{p}}$ between the $c$ and $a$ axes $\Delta \theta_{\mathrm{p}}$ from

$$
\Delta \theta_{\mathrm{p}}=\theta_{\mathrm{p}}^{c}-\theta_{\mathrm{p}}^{a}=-\frac{3(2 J-1)(2 J+3)}{10 k_{\mathrm{B}}} B_{2}^{0} .
$$

Assuming $J \approx 1$ from the effective moment determined from the inverse susceptibility, one can calculate $B_{2}^{0} / k_{\mathrm{B}}$ using Eq. (4) as $-10 \mathrm{~K}$ and $J_{e x}^{c} / k_{\mathrm{B}} \approx J_{e x}^{a} / k_{\mathrm{B}}=-674 \mathrm{~K}$. Here, a negative sign belongs to a ferromagnetic interaction. In first approximation the $B_{2}^{0}$ (usually $B_{2}^{0}$ is written with the symbol $D$ ) is related to $K_{1}$ by the relation $K_{1}=B_{2}^{0} J^{2} / V$, where $V$ is the volume per magnetic atom. Because $\mathrm{Rh}$ only provides a small induced spin moment without anisotropy, whereas Co provides both spin and orbital moments related to magnetocrystalline anisotropy, $V$ is roughly equal to the volume of the primitive cell. $K_{1}$ is estimated as $2.4 \mathrm{MJ} \mathrm{m}^{-3}$ from the above relation, which roughly agrees with the experimental value of $3.6 \mathrm{MJ} \mathrm{m}^{-3}$.

Since the crystal field is difficult to measure and, usually, $B_{2}^{0}$ is unknown, it is difficult to predict $\Delta \theta_{\mathrm{p}}$. However, below the Curie temperature, the crystal field will lead to magnetocrystalline anisotropy in ferromagnetic materials and, thus, we can roughly estimate $\Delta \theta_{\mathrm{p}}$ in the following way, assuming an isotropic exchange in ferromagnetic systems:

$$
\Delta \theta_{\mathrm{p}}=K_{1} V / k_{\mathrm{B}}
$$

Here, positive values of $K_{1}$ and $\Delta \theta_{\mathrm{p}}$ indicate the easy axis and a higher temperature along the $c$ axis, respectively. In $\mathrm{Rh}_{2} \mathrm{CoSb}$, the $K_{1}$ of $3.6 \mathrm{MJ} \mathrm{m}^{-3}$ is relatively large for a rare-earth free magnet. We obtained $\Delta \theta_{\mathrm{p}}=15 \mathrm{~K}$ from Eq. (5), which is exactly equal to the measured value, further confirming that the crystal field is the origin of all the above phenomena rather than the anisotropic exchange. We compared our theory with previous reports on some ferromagnetic materials in Fig. 5 and found that it works quite well. The slope of the curve is close to the Boltzmann constant $k_{\mathrm{B}}$.

The magnetocrystalline anisotropy varies from $10^{0}$ to $10^{4} \mathrm{~kJ} \mathrm{~m}^{-3}$ in ferromagnetic materials. Therefore, $\Delta \theta_{\mathrm{p}}$

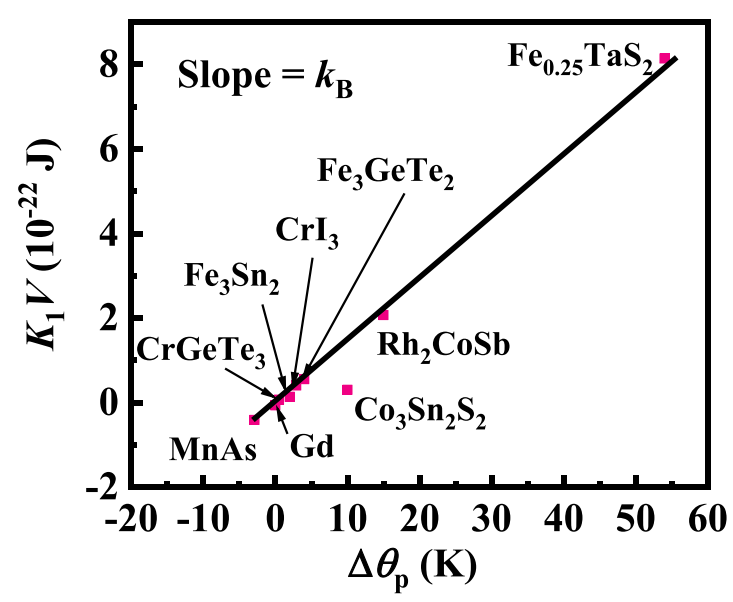

FIG. 5. Relationship between $K_{1} V$ and $\Delta \theta_{\mathrm{p}}$ for some ferromagnetic materials. References can be found in Table I.

calculated using Eq. (5) varies from $10^{-3}$ to $10^{1} \mathrm{~K}$. Assuming an isotropic exchange, $J_{e x}$, we can modify Eqs. (2) and (3) as

$$
\begin{aligned}
& \theta_{\mathrm{p}}^{c}=-\frac{J(J+1)}{3 k_{B}} J_{e x}+\frac{2}{3} K_{1} V / k_{B}, \\
& \theta_{\mathrm{p}}^{a}=-\frac{J(J+1)}{3 k_{B}} J_{e x}-\frac{1}{3} K_{1} V / k_{B} .
\end{aligned}
$$

From Eq. (7), there is a possibility that, for ferromagnets with an extremely large $K_{1}$ and a small exchange constant (low Curie temperature), $\theta_{\mathrm{p}}$ along the hard axis could even be negative, in contrast to the ferromagnetism. This has also been reported for some U-based compounds, such as $\mathrm{U}_{2} \mathrm{RhSi}_{3}$ [27], UPtAl [28], UIr [29], or $\mathrm{UCo}_{0.5} \mathrm{Sb}_{2}$ [30]. In these compounds, the anisotropy plays an even more important role than the exchange on $\theta_{\mathrm{p}}$ along the hard axis.

The calculated $\Delta \theta_{\mathrm{p}}$ works well for most ferromagnetic materials as shown in Table I. However, for antiferromagnetic/ferrimagnetic materials and magnetic materials with complicated spin structures, Eq. (5) is only valid for the sign of $\Delta \theta_{\mathrm{p}}$, indicating that the anisotropic exchange is dominant. In these cases, $\Delta \theta_{\mathrm{p}}$ can be as large as $100 \mathrm{~K}$, which is an order larger than the effect of the crystal field. This is one reason why previous reports on anisotropic $\Delta \theta_{\mathrm{p}}$ mainly focused on antiferromagnetic and ferrimagnetic materials.

Recently, an anomalous increase in the Curie temperature along the $c$ axis in ferromagnetic $\mathrm{CrTe}_{2}$ with decreasing thickness has been reported [43]. This is explained as the change from the easy-plane to the easy-axis spin structure, which further supports our theory that anisotropy plays an important role in the magnetic ordering temperature.

Of the anisotropic magnetic properties, magnetization is the easiest parameter to obtain. Therefore, compared with antiferromagnetic or ferrimagnetic materials whose magnetization is small, ferromagnetic materials show a much larger magnetization, whose signal can be detected and used in sensors for temperature measurement. Besides the temperature, the anisotropic critical temperature can also be used to detect the orientation. The difference in magnetization can be clearly observed close to or even above the Curie temperature. The ferromagnetic ordering can be turned on and off by changing 
TABLE I. Comparison of the anisotropic magnetization $\Delta m / m$ and paramagnetic Curie temperature $\Delta \theta_{\mathrm{p}}$ in different materials.

\begin{tabular}{|c|c|c|c|c|c|c|c|c|}
\hline Materials & Category & $K_{1}\left(\mathrm{MJ} \mathrm{m}^{-3}\right)$ & $\Delta m / m$ & $V, 10^{-30} \mathrm{~m}^{-3}$ & $\begin{array}{c}\text { Calculated } \\
\Delta \theta_{\mathrm{p}}(\mathrm{K})\end{array}$ & $\begin{array}{c}\text { Experiment } \\
\Delta \theta_{\mathrm{p}}(\mathrm{K})\end{array}$ & $\begin{array}{l}\text { Dominant } \\
\text { mechanism }\end{array}$ & References \\
\hline $\mathrm{Rh}_{2} \mathrm{CoSb}$ & Hard magnet & 3.6 & $25 \%$ & 58 & 15 & 15 & MCA & This paper \\
\hline $\mathrm{Fe}_{2} \mathrm{P}$ & Hard magnet & 2.5 & $9 \%$ & 17 & 3 & & $\mathrm{MCA}$ & {$[13]$} \\
\hline $\mathrm{Co}_{3} \mathrm{Sn}_{2} \mathrm{~S}_{2}$ & Hard magnet & 0.83 & $2 \%$ & 36 & 2 & 10 & $\mathrm{MCA}$ & [31] \\
\hline $\mathrm{Fe}_{3} \mathrm{Sn}_{2}$ & Frustrated magnet & 0.5 & $2 \%$ & 27 & 1 & 2 & MCA & {$[32,33]$} \\
\hline MnAs & Easy-plane & -1.2 & & 34 & -3 & -3 & MCA & [34] \\
\hline $\mathrm{Fe}_{3} \mathrm{GeTe}_{2}$ & 2D ferromagnet & 1.46 & $7 \%$ & 38 & 4 & 4 & $\mathrm{MCA}$ & {$[15]$} \\
\hline $\mathrm{CrI}_{3}$ & 2D ferromagnet & 0.3 & $3 \%$ & 135 & 3 & 3 & MCA & {$[16]$} \\
\hline $\mathrm{CrGeTe}_{3}$ & 2D ferromagnet & 0.05 & $1.6 \%$ & 137 & 0.5 & $<1$ & MCA & [35] \\
\hline $\mathrm{Fe}_{0.25} \mathrm{TaS}_{2}$ & 2D ferromagnet & 3.5 & & 230 & 58 & 54 & $\mathrm{MCA}$ & {$[36]$} \\
\hline $\mathrm{Fe}$ & Soft magnet & 0.04 & $0.015 \%$ & 13 & 0.04 & & $\mathrm{MCA}$ & {$[8]$} \\
\hline Ho & Helical (easy plane) & -66 & & 31 & -149 & -15 & MCA and EX & [40] \\
\hline $\mathrm{Er}$ & $c$-axis modulated & 10 & & 31 & 22 & 29 & MCA and EX & [41] \\
\hline $\mathrm{Tm}$ & $c$-axis modulated & 30 & & 30 & 65 & 58 & MCA and EX & {$[42]$} \\
\hline $\mathrm{Fe}_{7} \mathrm{~S}_{8}$ & FiM (easy plane) & -2.5 & & 33 & -6 & -378 & MCA and EX & {$[7]$} \\
\hline $\mathrm{CeAgSb}_{2}$ & Cycloidal & & & & & -63 & MCA and EX & [26] \\
\hline $\mathrm{TbRh}_{2} \mathrm{Si}_{2}$ & AFM (easy axis) & & & & & 120 & MCA and EX & [14] \\
\hline
\end{tabular}

Notes: MCA indicates magnetocrystalline anisotropy; EX indicates exchange; FiM and AFM indicate ferrimagnet and antiferromagnet, respectively. Here, the value of $K_{1}$ was used as the room-temperature value for MnBi. This category refers to the magnetic phase just below the magnetic ordering temperature. For heavy rare-earth elements, there are phases with ferromagnetic interactions at low temperatures. $\mathrm{Fe}_{2} \mathrm{P}, \mathrm{MnBi}$, and $\mathrm{MnAs}$ exhibit first-order transitions at the Curie temperature. $\mathrm{Fe}_{7} \mathrm{~S}_{8}$ has Fe vacancies on every second $a b$ plane, and the adjacent plans are antiferromagnetic coupled; therefore, it shows a net magnetic moment and behaves like a ferrimagnet with an in-plane applied magnetic field and an antiferromagnet with an applied out-of-plane field. Both $\Delta m$ and $\Delta \theta_{\mathrm{p}}$ for cubic Fe and Ni are calculated by the difference from the [001] and [111] directions, whose signs are the same as that of $K_{1}$ in cubic systems.

the crystal orientation in materials, such as $\mathrm{Rh}_{2} \mathrm{CoSb}$ with such a large anisotropic magnetic performance. Meanwhile, the anisotropic $\theta_{\mathrm{p}}$ can be used to estimate the $K_{1}$ for some materials that is extremely hard to saturate in the measurement.

\section{SUMMARY}

Extraordinary anisotropic magnetic properties have been reported for hard magnetic $\mathrm{Rh}_{2} \mathrm{CoSb}$. The magnetization along the $c$ axis is $25 \%$ larger than that along the $a$ axis. Different approaches to the magnetic phase transition are compared and used to describe the transition temperatures, including the critical temperature (to describe the long-range-ordered magnetic structure) and paramagnetic Curie temperatures (to describe the short-range-ordered magnetic structure). The critical temperature and paramagnetic Curie temperatures along the $c$ axis are 6 and $15 \mathrm{~K}$ higher than those along the $a$ axis, respectively, as deduced from the Arrott plots and inverse susceptibility. These values are some of the largest among ferromagnets without an anisotropic exchange. A simple modification of the paramagnetic Curie temperature is performed where the anisotropic value can be estimated from the magnetocrystalline anisotropy rather than from the complicated crystal-field parameters. The modification well explains not only $\mathrm{Rh}_{2} \mathrm{CoSb}$, but also many other previously reported ferromagnetic materials.

\section{ACKNOWLEDGMENTS}

This work was financially supported by an Advanced Grant from the European Research Council (Grant No. 742068) "TOPMAT," the European Union's Horizon 2020 Research and Innovation Programme (Grant No. 824123) "SKYTOP," the European Union's Horizon 2020 Research and Innovation Programme (Grant No. 766566) "ASPIN," the Deutsche Forschungsgemeinschaft (Project No. 258499086) "SFB 1143," the Deutsche Forschungsgemeinschaft (Projects No. FE 633/30-1, No. RE 1164/6-1, and No. LU 2261/2-1) "SPP Skyrmionics," the DFG through the Würzburg-Dresden Cluster of Excellence on Complexity and Topology in Quantum Matter ct.qmat (EXC 2147, Project No. 39085490). This work was based on experiments performed at the Swiss Spallation Neutron Source SINQ, Paul Scherrer Institute, Villigen, Switzerland.
[1] Y. He, X. Ke, C. Jiang, N. Miao, H. Wang, J. M. D. Coey, Y. Wang, and H. Xu, Adv. Funct. Mater. 28, 1800858 (2018).
[2] J. M. D. Coey, Magnetism and Magnetic Materials (Cambridge University Press, Cambridge, UK, 2010). 
[3] S. Yu. Bodnar, L. Šmejkal, I. Turek, T. Jungwirth, O. Gomonay, J. Sinova, A. A. Sapozhnik, H.-J. Elmers, M. Kläui, and M. Jourdan, Nat. Commun. 9, 348 (2018).

[4] E. R. Callen and H. B. Callen, J. Phys. Chem. Solids 16, 310 (1960).

[5] E. R. Callen, J. Appl. Phys. 31, S149 (1960).

[6] E. R. Callen, Phys. Rev. 124, 1373 (1961).

[7] J. N. Armstrong, S. Z. Hua, and H. D. Chopra, Phys. Status Solidi B 250, 387 (2013).

[8] Handbook of Magnetic Materials, edited by E. P Wohlfarth (North-Holland, Amsterdam, 1980), Vol. 1.

[9] J. M. Alameda, J. Deportes, D. Givord, R. Lemaire, and Q. Lu, J. Magn. Magn. Mater. 15, 1257 (1980).

[10] J. M. Alameda, D. Givord, R. Lemaire, and Q. Lu, J. Appl. Phys. 52, 2079 (1981).

[11] Y. He, S. Schneider, T. Helm, J. Gayles, D. Wolf, I. Soldatov, H. Borrmann, W. Schnelle, R. Schaefer, G. H. Fecher, B. Rellinghaus, and C. Felser, Topological Hall effect arising from the mesoscopic and microscopic non-coplanar magnetic structure in $\mathrm{MnBi}$, arXiv:2011.06340.

[12] M. A. McGuire, H. Cao, B. C. Chakoumakos, and B. C. Sales, Phys. Rev. B 90, 174425 (2014).

[13] L. Caron, M. Hudl, V. Höglin, N. H. Dung, C. P. Gomez, M. Sahlberg, E. Brück, Y. Andersson, and P. Nordblad, Phys. Rev. B 88, 094440 (2013).

[14] B. Chevalier, J. Etourneau, J. E. Greedan, J. M. D. Coey, and A. Maaroufi, J. Less-Common. Met. 111, 171 (1985).

[15] Y. Wang, C. Xian, J. Wang, B. Liu, L. Ling, L. Zhang, L. Cao, Z. Qu, and Y. Xiong, Phys. Rev. B 96, 134428 (2017).

[16] Y. Liu and C. Petrovic, Phys. Rev. B 97, 014420 (2018).

[17] P. Boutron, Phys. Rev. B 7, 3226 (1973).

[18] Y. L. Wang, Phys. Lett. A 35, 383 (1971).

[19] Y. He, G. H. Fecher, C. Fu et al., Adv. Mater. 32, 2004331 (2020).

[20] J. Rodríguez-Carvajal, Commission on powder diffraction (IUCr), Newsletter 26, 12 (2001).

[21] G. H. Fecher, Y. He, and C. Felser, Phys. Rev. Materials 5, 054404 (2021).

[22] S. K. Dhar, A. K. Grover, S. K. Malik, and R. Vijayaraghavan, Solid State Commun. 33, 545 (1980).

[23] N. J. Ghimire, M. A. McGuire, D. S. Parker, B. C. Sales, J.-Q. Yan, V. Keppens, M. Koehler, R. M. Latture, and D. Mandrus, Phys. Rev. B 85, 224405 (2012).
[24] M. K. Chattopadhyay, P. Arora, and S. B. Roy, J. Phys. Condens. Matter. 21, 296003 (2009).

[25] J. Fan, L. Ling, B. Hong, L. Zhang, L. Pi, and Y. Zhang, Phys. Rev. B 81, 144426 (2010).

[26] K. D. Myers, S. L. Bud'ko, I. R. Fisher, Z. Islam, H. Kleinke, A. H. Lacerda, and P. C. Canfield, J. Magn. Magn. Mater. 205, 27 (1999).

[27] M. Szlawska, M. Majewicz, and D. Kaczorowski, J. Alloys Compd. 662, 208 (2016).

[28] A. V. Andreev, Y. Shiokawa, M. Tomida, Y. Homma, V. Sechovský, N. V. Mushnikov, and T. Goto, J. Phys. Soc. Jpn. 68, 2426 (1999).

[29] A. Galatanu, Y. Haga, E. Yamamoto, T. D. Matsuda, S. Ikeda, and Y. Ōnuki, J. Phys. Soc. Jpn. 73, 766 (2004).

[30] Z. Bukowski, V. H. Tran, J. Stepień-Damm, and R. Troć, J. Solid State Chem. 177, 3934 (2004).

[31] J. Shen, Q. Zeng, S. Zhang, W. Tong, L. Ling, C. Xi, Z. Wang, E. Liu, W. Wang, G. Wu, and B. Shen, Appl. Phys. Lett. 115, 212403 (2019).

[32] H. Li, B. Ding, J. Chen, Z. Li, Z. Hou, E. Liu, H. Zhang, X. Xi, G. Wu, and W. Wang, Appl. Phys. Lett. 114, 192408 (2019).

[33] Q. Wang, S. Sun, X. Zhang, F. Pang, and H. Lei, Phys. Rev. B 94, 075135 (2016).

[34] R. W. De Blois and D. S. Rodbell, Phys. Rev. 130, 1347 (1963).

[35] S. Selter, G. Bastien, A. U. B. Wolter, S. Aswartham, and B. Büchner, Phys. Rev. B 101, 014440 (2020).

[36] E. Morosan, H. W. Zandbergen, L. Li, M. Lee, J. G. Checkelsky, M. Heinrich, T. Siegrist, N. P. Ong, and R. J. Cava, Phys. Rev. B 75, 104401 (2007).

[37] H. E. Nigh, S. Legvold, and F. H. Spedding, Phys. Rev. 132, 1092 (1963).

[38] D. E. Hegland, S. Legvold, and F. H. Spedding, Phys. Rev. 131, 158 (1963).

[39] D. R. Behrendt, S. Legvold, and F. H. Spedding, Phys. Rev. 109, 1544 (1985).

[40] D. L. Strandburg, S. Legvold, and F. H. Spedding, Phys. Rev. 127, 2046 (1962).

[41] R. W. Green, S. Legvold, and F. H. Spedding, Phys. Rev. 122, 827 (1961).

[42] D. B. Richards and S. Legvold, Phys. Rev. 186, 508 (1969).

[43] L. Meng, Z. Zhou, M. Xu, S. Yang, K. Si, L. Liu, X. Wang, H. Jiang, B. Li, P. Qin, P. Zhang, J. Wang, Z. Liu, P. Tang, Y. Ye, W. Zhou, L. Bao, H. Gao, and Y. Gong, Nat. Commun. 12, 809 (2021). 